\title{
Osteoporosis, osteopenia, and atherosclerotic vascular disease
}

Wilbert S. Aronow

Divisions of Cardiology, Geriatrics, and Pulmonary/Critical Care, Department of Medicine, New York Medical College, Valhalla, New York, USA

Submitted: 11 August 2010

Accepted: 12 August 2010

Arch Med Sci 2011; 7, 1: 21-26

DOI: 10.5114/aoms.2011.20599

Copyright () 2011 Termedia \& Banach

\section{Abstract}

Older women with low bone mineral density (BMD) have a higher prevalence of atherosclerotic vascular disease (coronary artery disease, ischemic stroke, or peripheral arterial disease) than older women with normal BMD. Three coronary angiographic studies have shown that low BMD is associated with obstructive coronary artery disease. Low BMD has been shown to be associated with stress test-induced myocardial ischemia, reduced exercise capacity, and with aortic valve calcification. Women with osteoporosis have an increased risk for cardiovascular events. Treatment of osteoporosis or osteopenia should include therapeutic measures to prevent cardiovascular events.

Key words: osteoporosis, osteopenia, bone mineral density, coronary artery disease, myocardial ischemia, atherosclerotic vascular disease.

\section{Introduction}

According to the criteria of the World Health Organization, osteoporosis is diagnosed when the bone mineral density (BMD) is greater than 2.5 standard deviations below peak bone mass and osteopenia is diagnosed when the BMD is 1-2.5 standard deviations below peak bone mass [1]. This review article will discuss the relationship between low BMD and atherosclerotic vascular disease.

\section{Atherosclerotic vascular disease}

Dual energy X-ray absorptiometry (DEXA) scans were used to measure $B M D$ in a retrospective case comparison pilot study in 101 postmenopausal women, mean age 76 years, in a nursing home affiliated with New York Medical College [2]. The DEXA scans were obtained because the United States Preventive Services Task Force and the National Osteoporosis Foundation recommend measuring BMD at least once in all women older than 65 years $[3,4]$. BMD was measured in the hips and spine in all 101 patients by DEXA scan obtained from a computerized radiology database. Of 101 patients, 51 (51\%) had a low BMD [2].

The mean age and prevalence of smoking, hypertension, diabetes mellitus, and hypercholesterolemia were not significantly different between women with a low BMD and those with a normal BMD. However, atherosclerotic vascular disease defined as symptomatic coronary artery disease, cerebrovascular disease, or peripheral arterial disease was present

\author{
Corresponding author: \\ Wilbert S. Aronow, MD \\ Divisions of Cardiology \\ Geriatrics, and \\ Pulmonary/Critical Care \\ Department of Medicine \\ New York Medical College \\ Macy Pavilion, Room 138 \\ Valhalla \\ NY 10595 USA \\ Phone: +914 4935311 \\ Fax: +914 2356274 \\ E-mail:wsaronow@aol.com
}


in 31 of 51 women (61\%) with osteoporosis or osteopenia versus 19 of 38 women (38\%) with normal BMD $(p<0.025)$ [2]. Coronary artery disease and cerebrovascular disease were diagnosed as previously described [5-8].

A retrospective analysis was performed in 1000 postmenopausal women, mean age 68 years (range 51 to 97 years), seen consecutively at a general medicine clinic at the University of Iowa School of Medicine to investigate the prevalence of atherosclerotic vascular disease in women with osteoporosis, osteopenia, and no osteoporosis or osteopenia [9]. Women were diagnosed as having osteoporosis or osteopenia according to the criteria of the World Health Organization [1].

Of 1000 women, 154 (15\%) had osteoporosis, 179 (18\%) had osteopenia, and 667 (67\%) had no osteoporosis or osteopenia [9]. The mean age and prevalence of race, current cigarette smoking, hypertension, diabetes mellitus, and hypercholesterolemia were not significantly different between women with osteoporosis, osteopenia, and no osteoporosis or osteopenia. Atherosclerotic vascular disease was defined as the presence of coronary artery disease, ischemic stroke, or peripheral arterial disease. Atherosclerotic vascular disease occurred in 92 of 154 women (60\%) with osteoporosis, in 63 of 179 women (35\%) with osteopenia, and in 148 of 667 women (22\%) with no osteoporosis or osteopenia. $P$ values were $<0.001$ comparing osteoporosis with osteopenia, $<0.001$ comparing osteoporosis with no osteoporosis or osteopenia, and < 0.001 comparing osteopenia with no osteoporosis or osteopenia [9].

In 31936 Swedish twins followed up from age 50 years, the adjusted hazard ratio for hip fracture was significantly increased 4.40 times after a diagnosis of heart failure, 5.09 times after a diagnosis of stroke, 3.20 times after a diagnosis of peripheral arterial disease, and 2.32 times after a coronary event [10].

\section{Coronary artery disease}

In 5050 women and men aged 50 to 79 years, self-reported myocardial infarction was insignificantly increased by $22 \%$ in women with a low BMD and was significantly increased $(p=0.03)$ by $39 \%$ in men with a low BMD [11]. In 45 postmenopausal women who had DEXA scans and coronary artery calcium scores measured, the latter were 42 for women with a normal BMD, 182 for women with osteopenia, and 222 for women with osteoporosis, suggesting that women with a low BMD are at higher risk of coronary atherosclerosis [12].

Using the Framingham coronary heart disease risk prediction algorithm in 1804 women aged 50 to 74 years, women with a $10 \%$ to $19 \%$ coronary heart disease risk within 10 years were significantly
$45 \%$ more likely to have a low BMD than women with a less than $10 \%$ chance of developing coronary heart disease within 10 years [13]. Women with a $20 \%$ or higher coronary heart disease risk within 10 years were significantly $73 \%$ more likely to have a low BMD than women with a less than $10 \%$ chance of developing coronary heart disease within 10 years. Similar increases in low BMD risk were not observed in men [13].

Marcovitz et al. showed in 209 patients (89\% women and $11 \%$ men), mean age 67 years, that obstructive coronary artery disease with $\geq 50 \%$ narrowing of at least 1 major coronary artery was present in 122 of 157 patients (78\%) with a low BMD and in 26 of 52 patients (50\%) with a normal BMD ( $p<0.001)$ [14]. In this study, multivessel coronary artery disease was present in 86 of 157 patients (55\%) with a low BMD and in 13 of 52 patients $(25 \%)$ with a normal BMD $(p=0.0002)$. The DEXA scans and coronary angiograms were performed within the same 12-month period.

Multivariate step-down logistic regression analysis showed that significant independent predictors of obstructive coronary artery disease were osteoporosis (odds ratio $[\mathrm{OR}]=5.58$ ), hypertension ( $O R=3.92)$, family history of premature coronary artery disease $(O R=2.99)$, and fasting glucose level greater than $110 \mathrm{mg} / \mathrm{dl}$ $(\mathrm{OR}=3.28)$ [14]. Osteoporosis and osteopenia were also significantly associated with any degree of vessel narrowing [14].

Coronary angiography was performed because of chest pain in 198 patients ( $76 \%$ women and $24 \%$ men), mean age 66 years, who had DEXA scans of the spine and left hip because of suspected osteoporosis or osteopenia within 6 months of coronary angiography [15]. Osteoporosis was diagnosed when the T score was $<-2.5$ standard deviations below peak bone mass and osteopenia was diagnosed when the T score was -1.0 to -2.5 standard deviations below peak bone mass [16]. Normal BMD was diagnosed if the T score was $>-1.0$ standard deviations below peak bone mass [16]. Obstructive coronary artery disease was diagnosed if there was > 50\% diameter narrowing of at least 1 major coronary artery.

Of 198 patients, 53 (27\%) had osteoporosis, 79 (40\%) had osteopenia, and 66 (33\%) had normal BMD. The prevalence of gender, mean age, prior myocardial infarction, current smoking, hypertension, diabetes mellitus, hypercholesterolemia, and body mass index $\geq 30 \mathrm{~kg} / \mathrm{m}^{2}$ were not significantly different between patients with osteoporosis, osteopenia, and normal BMD. Obstructive coronary artery disease with > 50\% narrowing of at least 1 major coronary artery was present in 40 of 53 patients (76\%) with osteoporosis, in 54 of 79 patients (68\%) with 
osteopenia, and in 31 of 66 patients (47\%) with normal BMD ( $p<0.005$ comparing osteoporosis with normal BMD and $p<0.01$ comparing osteopenia with normal BMD) [15].

The prevalence of obstructive coronary artery disease was significantly higher in women with osteoporosis (74\%) or osteopenia (66\%) than in women with normal BMD (45\%) [15]. The prevalence of obstructive coronary artery disease was $80 \%$ in men with osteoporosis, $75 \%$ in men with osteopenia, and 53\% in men with normal BMD [15]. These differences were not significantly different because of the small number of men included in the study.

These data confirmed the association of obstructive coronary artery disease with osteoporosis and osteopenia reported by Marcovitz et al. [14]. A retrospective analysis of 47 men, mean age 65 years, who had DEXA scans and coronary angiography showed that low BMD was an independent predictor of obstructive coronary artery disease with an odds ratio of $5.4(95 \% \mathrm{Cl}, 1.66$ to 17.49) [17]. The mechanism of this association remains to be investigated.

\section{Osteopontin}

Osteopontin is a noncollagenous bone matrix protein produced by osteocytes, osteoblasts, and osteoclasts that can interact with the cell surface receptor CD $44[18,19]$. Osteopontin is associated with the presence and extent of coronary artery disease [20]. In 178 patients undergoing coronary angiography, mean age 65 years, plasma osteopontin levels were significantly associated with coronary artery disease, independent of other risk factors [20]. Coronary artery calcification was present in 86 of 178 patients (48\%). Plasma osteopontin levels were higher in patients with coronary artery calcification than in patients without coronary artery calcification $(p<0.01)$ and correlated with the number of calcified segments $(p<0.001)$, but were not independently associated with coronary artery calcification [20].

Production of osteopontin by circulating endothelial progenitor cells at sites of tissue injury may modulate proliferation, migration, and accumulation of endothelial and vascular smooth muscle cells, promoting vascular repair but initiating vascular calcification [19, 21, 22]. The same proinflammatory factors involved in the pathogenesis of osteoporosis may lead to expression of an osteogenic phenotype by endothelial lineage cells, providing a potential mechanism for the association between osteoporosis and coronary artery disease [19].

Observational data support the association of osteopontin with atherosclerotic and valvular heart disease [23]. An increased osteoprotegerin level may be an indicator of a proinflammatory milieu that promotes atherosclerosis [24].

\section{Exercise stress testing}

Exercise capacity measured by exercise stress testing is a predictor of coronary mortality independent of traditional risk factors for coronary artery disease [25-27]. Showing an association between BMD and exercise capacity could help explain the association between BMD and coronary artery disease [28]. Therefore, From et al. studied a cohort of patients who had undergone both exercise echocardiography and DEXA scans to determine whether patients with low BMD had lower exercise capacities and were more likely to have exercise-induced ischemic changes during exercise echocardiography than patients with normal BMD [28].

Of 1142 patients, mean age 64 years, included in this study, 1001 (88\%) were women and 141 (12\%) were men [24]. Of the 1142 patients, 126 (11\%) had osteoporosis, 517 (45\%) had osteopenia, and 499 (44\%) had normal BMD. Reasons for stopping the exercise echocardiographic stress test were fatigue in 761 patients $(67 \%)$, dyspnea in 273 patients (24\%), hypertension in 66 patients (6\%), leg distress in 46 patients (4\%), angina pectoris in 15 patients (1\%), completion of protocol in 18 patients (2\%), and reduction of blood pressure in 10 patients (1\%).

The treadmill exercise duration was $7.3 \mathrm{~min}$ in patients with a low BMD versus $8.1 \mathrm{~min}$ in patients with a normal BMD $(p<0.001)$ [28]. Myocardial ischemia developed during exercise echocardiography in $21 \%$ of patients with low BMD versus $14.7 \%$ of patients with normal BMD $(p=0.007)$. Patients with normal BMD had lower wall motion score indexes during exercise echocardiography than patients with low BMD ( $p<0.001$ by Wilcoxon rank sum test).

For every 1-unit reduction in femoral neck T score, a 0.23 min significant reduction in treadmill exercise duration was found after values were adjusted for age and other patient characteristics. For every 1-unit reduction in femoral neck T score, there was a significant $22 \%$ increased risk of myocardial ischemia after values were adjusted for age and other patient characteristics. Overall after adjustments, patients with a low BMD who were referred for exercise echocardiographic stress testing, had a $43 \%$ significantly greater risk of myocardial ischemia, than did patients with normal BMD [28].

This study was the first study to demonstrate that low BMD is associated with exercise-induced myocardial ischemia and with reduced exercise capacity [28]. From et al. stated that the association between low BMD and reduced exercise capacity suggests that reduced physical activity may in part 
explain the relationship between low BMD and myocardial ischemia [28]. These findings support the studies that have linked increased mortality and higher bone turnover to reduced physical activity $[29,30]$. From et al. suggested that reduced physical activity contributes to both low BMD and coronary artery disease through the development of atherosclerotic vascular disease [28]. Reduced exercise capacity and reduced physical activity are well established predictors of coronary artery disease and mortality [25-27].

These findings are strengthened by research findings demonstrating that various proteins known to regulate bone formation and bone resorption, including osteoprotegerin, matrix Gla protein, bone morphogenetic protein and osteopontin, are present in calcified atherosclerotic lesions [31].

DEXA scans of the spine and left hip were obtained prior to stress testing for myocardial ischemia in 629 women (82\%) and 136 men (18\%), mean age 63 years, with chest pain typical of ischemia and no prior history of coronary artery disease [32]. No patients with BMD measurements were excluded from this study. Of the 765 patients, $342(45 \%)$ had an adenosine sestamibi or dipyridamole stress test, $336(44 \%)$ had a treadmill sestamibi stress test, $80(10 \%)$ had a treadmill stress test, and 7 (1\%) had an exercise stress echocardiogram. A treadmill stress test was considered positive if ST-segment depression developed at least $1.0 \mathrm{~mm}$ below the resting level, with the ST segment extending horizontally for at least $80 \mathrm{msec}$ after the J point or with downsloping of the ST-segment. In the presence of resting ST-segment depression, an increase of at least $2.0 \mathrm{~mm}$ below the resting level was required [33]. Stress tests were analyzed for the presence of myocardial ischemia without knowledge whether the patient had osteoporosis, osteopenia, or normal BMD.

Osteoporosis was diagnosed if the T score was $<-2.5$ standard deviations below peak bone mass [16]. Osteopenia was diagnosed if the T score was -1.0 to -2.5 standard deviations below peak bone mass [16]. Normal BMD was diagnosed if the T score was $>-1.0$ standard deviations below peak bone mass [16].

Of the 765 patients, 254 (33\%) had osteoporosis, 260 (34\%) had osteopenia, and 251 (33\%) had normal BMD [32]. The mean age and prevalence of gender, hypertension, hypercholesterolemia, diabetes mellitus, current smoking, body mass index $\geq 30 \mathrm{~kg} / \mathrm{m}^{2}$, and estimated glomerular filtration rate $<60 \mathrm{ml} / \mathrm{min} / 1.73 \mathrm{~m}^{2}$ were not significantly different between patients with osteoporosis, osteopenia, and normal BMD.

Stress test-induced myocardial ischemia developed in 95 of 254 patients (37\%) with osteoporosis, in 81 of 260 patients (31\%) with osteopenia, and in 62 of 251 patients (25\%) with normal BMD ( $p=0.009)$ ( $p=0.002$ comparing osteoporosis with normal BMD; $p=0.007$ comparing osteoporosis or osteopenia with normal BMD) [32]. The increased prevalence of myocardial ischemia in patients with low BMD was observed for both exercise and pharmacological stress testing.

Stepwise logistic regression analysis to identify significant independent risk factors for stress testinduced myocardial ischemia were hypertension $(\mathrm{OR}=1.7)$, diabetes mellitus $(\mathrm{OR}=2.3)$, body mass index $\geq 30 \mathrm{~kg} / \mathrm{m}^{2}(\mathrm{OR}=1.6)$, age $(\mathrm{OR}=1.02$ per year increase in age), and low BMD. Patients with osteoporosis or osteopenia had a 1.7 times higher chance of stress test-induced myocardial ischemia than those with normal BMD after controlling the confounding effects of hypertension, diabetes mellitus, body mass index, and age [32]. These data confirmed the association between low BMD and stress test-induced myocardial ischemia reported by From et al. [28].

Tanko et al. found in 2576 women, mean age 67 years, in the placebo group of the Multiple Outcomes of Raloxifene Evaluation (MORE) randomized study that the risk of cardiovascular events significantly increased incrementally with the number and increasing severity of baseline vertebral fractures [34]. After adjustment for potential confounders, women with osteoporosis had a 3.9 times significantly increased risk for cardiovascular events compared with women with low bone mass [34]. These investigators recommended that treatment of postmenopausal osteoporosis should include consideration of measures to prevent cardiovascular events.

\section{Aortic valve calcification}

An association between low BMD and increased prevalence of aortic valve calcification [35] and of abdominal aortic calcifications [36] has been observed in older women with low BMD. Skolnick et al. also demonstrated in 55 patients with osteoporosis and aortic stenosis that treatment of osteoporosis was associated with a significant reduction in progression of aortic stenosis at 2.4-year follow-up [37]. These data warrant further investigation.

\section{Mechanisms}

As discussed, mechanisms for the association between low BMD and atherosclerotic vascular disease and valvular calcification include common risk factors including smoking, hypertension, physical inactivity, dyslipidemia, and menopause. Increased plasma osteopontin levels and proinflammatory factors as discussed also play a role. 
Osteoprotegerin-deficient mice develop early onset osteoporosis and calcification of the aorta and renal arteries [38]. An atherogenic high fat diet reduces bone formation in mice [39]. Use of statins has been associated with higher BMD [40] and decreased risk of fractures [41, 42]. Mice lacking the gene for matrix gla protein have vascular calcification, osteopenia, and fractures [43]. The ApoE 4 gene is also associated with decreased BMD and increased risk of fractures $[44,45]$.

In conclusion, on the basis of the available findings, patients with osteoporosis or osteopenia should be considered of high cardiovascular risk, especially when other major cardiovascular risk factors coexist. Exercise stress testing could be the diagnostic method of choice to rule out coronary artery disease in these patients. Patients with osteoporosis or osteopenia should also be treated with intensive risk factor modification in addition to treatment of the osteoporosis or osteopenia. Further studies are indicated to investigate the mechanisms by which low BMD is associated with coronary artery disease, stress test-induced myocardial ischemia, atherosclerotic vascular disease, and aortic valve calcification.

\section{References}

1. World Health Organization. Guidelines for Preclinical Evaluation and Clinical Trials in Osteoporosis. World Health Organization, Geneva 1998.

2. Gupta G, Aronow WS. Atherosclerotic disease may be associated with osteoporosis or osteopenia in postmenopausal women: a preliminary study. Arch Gerontol Geriatr 2006; 43: 285-8.

3. Preventive Services Task Force. Screening for osteoporosis in postmenopausal women: recommendations and rationale. Ann Intern Med 2002; 137: 526-8.

4. National Osteoporosis Foundation. Physician's guide to prevention and treatment of osteoporosis. National Osteoporosis Foundation, Washington 2003.

5. Lai HM, Aronow WS, Rachdev A, et al. Incidence of mortality in 1,040 patients with coronary heart disease or hypertensive heart disease with normal and abnormal left ventricular ejection fraction and with normal and abnormal QRS duration. Arch Med Sci 2008; 4: 140-2.

6. Kannam H, Aronow WS, Chilappa K, et al. Association of the QRS duration on the resting electrocardiogram with the severity of coronary artery disease in 2,196 patients undergoing coronary angiography for suspected coronary artery disease. Arch Med Sci 2009; 5: 163-5.

7. Lleva P, Aronow WS, Amin H, et al. Prevalence of electrocardiographic abnormalities in patients with ischemic stroke, intracerebral hemorrhage, and subarachnoid hemorrhage. Arch Med Sci 2008; 4: 259-62.

8. Amin $\mathrm{H}$, Aronow WS, Lleva $\mathrm{P}$, et al. Prevalence of transthoracic echocardiographic abnormalities in patients with ischemic stroke, intracerebral hemorrhage, and subarachnoid hemorrhage. Arch Med Sci 2010; 6: 40-2.

9. Ness J, Aronow WS. Comparison of prevalence of atherosclerotic vascular disease in postmenopaual women with osteoporosis or osteopenia versus without osteoporosis or osteopenia. Am J Cardiol 2006; 97: 1427-8.
10. Sennerby U, Melhus H, Gedeborg R, et al. Cardiovascular diseases and risk of hip fracture. JAMA 2009; 302: 1666-73.

11. Magnus JH, Brussard DL. Relationship between bone mineral density and myocardial infarction in US adults. Osteoporosis Int 2005; 16: 2053-62.

12. Barengolts El, Berman M, Kukreja SC, et al. Osteoporosis and coronary atherosclerosis in asymptomatic postmenopausal women. Calcif Tissue Int 1998; 62: 209-13.

13. Broussard DL, Magnus JH. Coronary heart disease risk and bone mineral density among U.S. women and men. J Women's Health 2008; 17: 479-90.

14. Marcovitz PA, Tran HH, Franklin BA, et al. Usefulness of bone mineral density to predict significant coronary artery disease. Am J Cardiol 2005; 96: 1059-63.

15. Varma R, Aronow WS, Basis Y, et al. Relation of bone mineral density to frequency of coronary heart disease. Am J Cardiol 2008; 101: 1103-4.

16. Genant HK, Cooper C, Reid I, et al. Interim report and recommendations of the World Health organization Task force for Osteoporosis. Osteoporosis Int 1999; 10: 259-64.

17. Erbilen E, Yazici S, Ozhan $\mathrm{H}$, et al. Relationship between angiographically documented coronary artery disease and low bone mass in men. Circ J 2007; 7: 1095-8.

18. Weber GF, Ashkar S, Glimcher MJ, Cantor H. Receptorligand interaction between CD44 and osteopontin (Eta-1). Science 1996; 271: 509-12.

19. Gossl M, Modder UI, Atkinson EJ, et al. Osteocalcin expression by circulating endothelial progenitor cells in patients with coronary atherosclerosis. J Am Coll Cardiol 2008; 52: 1314-25.

20. Ohmori R, Momiyama Y, Taniguchi $\mathrm{H}$, et al. Plasma osteopontin levels are associated with the presence and extent of coronary artery disease. Atherosclerosis 2003; 170: 333-7.

21. Liaw L, Almeida M, Hart CE, et al. Osteopontin promotes vascular cell adhesion and spreading and is chemotactic for smooth muscle cells in vitro. Circ Res 1994; 74: 214-24.

22. Standal T, Borset M, Sundan A. Role of osteopontin in adhesion, migration, cell survival and bone remodeling. Exp Oncol 2004; 26: 179-84.

23. Waller AH, Sanchez-Ross M, Kaluski E, Klapholz M. Osteopontin in cardiovascular disease. A potential therapeutic target. Cardiol Rev 2010; 18: 125-31.

24. Venuraju SM, Yerramasu A, Corder R, Lahiri A. Osteoprotegerin as a predictor of coronary artery disease and cardiovascular mortality and morbidity. J Am Coll Cardiol 2010; 55: 2049-61.

25. Arruda AM, Das MK, Roger VL, et al. Prognostic value of exercise echocardiography in 2,632 patients $\geq 65$ years of age. J Am Coll Cardiol 2001; 37: 1036-41.

26. Peterson PN, Magid DJ, Ross C, et al. Association of exercise capacity on treadmill with future cardiac events in patients referred for exercise testing. Arch Intern Med 2008; 168: 174-9.

27. Pierre-Louis B, Aronow WS, Yoon JH, et al. Incidence of myocardial infarction or stroke or death at 47-month follow-up in patients with diabetes and a predicted exercise capacity less than $85 \%$ versus greater than $85 \%$ during an exercise treadmill sestamibi stress test. Preventive Cardiology 2010; 13: 14-7.

28. From AM, Hyder JA, Kearns AE, et al. Relationship between low bone mineral density and exercise-induced myocardial ischemia. Mayo Clin Proc 2007; 82: 679-85.

29. Greg EW, Cauley JA, Stone K, et al. Study of Osteoporotic Fractures Research Group. Relationship of changes in 
physical activity and mortality among older women. JAMA 2003; 289: 2379-86.

30. Chen JS, Cameron ID, Cumming RG, et al. Effect of agerelated chronic immobility on markers of bone turnover. J Bone Miner Res 2006; 21: 324-31.

31. Abedin M, Tintut Y, Demer LL. Vascular calcification: mechanisms and clinical ramifications. Arterioscler Thromb Vasc Biol 2004; 24: 1161-70.

32. Pierre-Louis B, Aronow WS, Yoon JH, et al. Relation of bone mineral density to stress test-induced myocardial ischemia. Am J Cardiol 2009; 104: 199-201.

33. DeLuca AJ, Kaplan S, Aronow WS, et al. Comparison of prevalence of unrecognized myocardial infarction and of silent myocardial ischemia detected by a treadmil exercise sestamibi stress test in patients with versus without diabetes mellitus. Am J Cardiol 2006; 98: 1045-6.

34. Tanko LB, Christiansen C, Cox DA, et al. Relationship between osteoporosis and cardiovascular disease in postmenopausal women. J Bone Miner Res 2005; 20 1912-20.

35. Aksoy Y, Yagmur C, Tekin GO, et al. Aortic valve calcification: association with bone mineral density and cardiovascular risk factors. Coron Artery Dis 2005; 16: 379-83.

36. Jie KG, Bots ML, Vermeer C, et al. Vitamin K status and bone mass in women with and without aortic atherosclerosis: a population-based study. Calcif Tissue Int 1996; 59: 352-6.

37. Skolnick AH, Osranek M, Formica P, Kronzon I. Osteoporosis treatment and progression of aortic stenosis. Am J Cardiol 2009; 104: 122-4.

38. Bucay N, Sarosi I, Dunstan CR, et al. Osteoprotegerindeficient mice develop early onset osteoporosis and arterial calcification. Genes Dev 1998; 12: 1260-8.

39. Parhami F, Tintut Y, Beamer WG, et al. Atherogenic highfat diet reduces bone mineralization in mice. JBMR 2001; 16: $182-8$.

40. Solomon DH, Finkelstein JS, Wang P, Avorn J. Statin lipid-lowering drugs and bone mineral density. Pharmacoepidemiol Drug Saf 2005; 14: 219-26.

41. Bauer DC, Mundy GR, Jamal SA, et al. Use of statins and fracture. Results of 4 prospective studies and cumulative meta-analysis of observational studies and controlled trials. Arch Intern Med 2004; 164: 146-52.

42. Pasco JA, kotowicz MA, Henry MJ, et al. Statin use, bone mineral density, and fracture risk. Arch Intern Med 2002; 162: $537-40$

43. Luo G, Ducy P, McKee MD, et al. Spontaneous calcification of arteries and cartilage in mice lacking matrix GLA protein. Nature 1997; 386: 78-81

44. Shiraki M, Shiraki Y, Aoki C, et al. Association of bone mineral density with apolipoprotein E phenotype. J Bone Min Res 1997; 12: 1438-45.

45. Cauley JA, Zmuda JM, Yaffe K, et al. Apolipoprotein E polymorphism : a new genetic marker of hip fracture risk - The Study of Osteoporotic Fractures. J Bone Min Res 1999; 14: 1175-81. 